\title{
Cytokinesis Depends on the Motor Domains of Myosin-II in Fission Yeast but Not in Budding Yeast
}

\author{
Matthew Lord, ${ }^{*}$ Ellen Laves, ${ }^{*}$ and Thomas D. Pollard ${ }^{*+\ddagger}$
}

\author{
Departments of *Molecular, Cellular, and Developmental Biology, ${ }^{+}$Molecular Biophysics and Biochemistry, \\ and ¥Cell Biology, Yale University, New Haven, CT 06520-8103
}

Submitted July 6, 2005; Revised August 19, 2005; Accepted August 25, 2005

Monitoring Editor: Anthony Bretscher

Budding yeast possesses one myosin-II, Myo1p, whereas fission yeast has two, Myo2p and Myp2p, all of which contribute
to cytokinesis. We find that chimeras consisting of Myo2p or Myp2p motor domains fused to the tail of Myo1p are fully
functional in supporting budding yeast cytokinesis. Remarkably, the tail alone of budding yeast Myo1p localizes to the
contractile ring, supporting both its constriction and cytokinesis. In contrast, fission yeast Myo2p and Myp2p require both
the catalytic head domain as well as tail domains for function, with the tails providing distinct functions (Bezanilla and
Pollard, 2000). Myo1p is the first example of a myosin whose cellular function does not require a catalytic motor domain
revealing a novel mechanism of action for budding yeast myosin-II independent of actin binding and ATPase activity.

\section{INTRODUCTION}

Cytokinesis, the physical separation of dividing cells, is a fundamental but poorly understood event. Formation of a cleavage furrow depends on the actin-based motor myosin-II to constrict a contractile ring of actin filaments (Mabuchi and Okuno, 1977). Genetic studies in budding and fission yeast (Guertin et al., 2002) and RNA interference experiments on animal cells (Somma et al., 2002; Kiger et al., 2003; Rogers et al., 2003; Echard et al., 2004) have identified a growing list of other genes required for cytokinesis.

The heavy chain of myosin-II forms an N-terminal catalytic domain (head) and a C-terminal coiled-coil tail. The head binds actin filaments and catalyzes ATP hydrolysis. The segment of the heavy chain between the catalytic domain and the tail binds two light chains related to calmodulin: an essential light chain (ELC) and a regulatory light chain (RLC). This light chain domain forms a lever arm that amplifies the force-producing conformational changes in the head. Phosphorylation of RLCs regulates the motor activity of animal cell myosin-II (Sellers et al., 1981; Bresnick, 1999), whereas an Unc45-/Cro1p-/She4p-related (UCS) protein regulates the motor activity of fission yeast myosin-II (Lord and Pollard, 2004). Myosin-II tails assemble into bipolar filaments, allowing contraction of actin filaments in muscle and other cells. The tails of nonmuscle myosin-II may have additional functions. The tails of myosin-II from Dictyostelium and Acanthamoeba have a role in recruitment to the cleavage furrow (Yumura and Uyeda, 1997; Zang and Spudich, 1998; Shu et al., 1999, 2003).

The fission yeast, Schizosaccharomyces pombe, has two myosin-II heavy chains, both paired with ELC Cdc4p (McCollum et al., 1995; Motegi et al., 2000; Naqvi et al., 2000; Lord et

This article was published online ahead of print in $M B C$ in Press (http:/ / www.molbiolcell.org/cgi/doi/10.1091/mbc.E05-07-0601) on September 7, 2005.

Address correspondence to: Thomas D. Pollard (thomas.pollard@ yale.edu).

Abbreviations used: ELC, essential light chain; RLC, regulatory light chain; UCS, Unc-45-/Cro1p-/She4p-related. al., 2004) and RLC Rlc1p (Le Goff et al., 2000; Naqvi et al., 2000; Lord and Pollard, 2004). Myo2p is essential for cytokinesis and viability under most conditions (Kitayama et al., 1997; May et al., 1997; Balasubramanian et al., 1998), but Myp2p is required only under special stressful conditions (Bezanilla et al., 1997; Motegi et al., 1997). The tails specify unique functions, including localization of Myo2p and Myp2p at the contractile ring (Naqvi et al., 1999; Bezanilla and Pollard, 2000; Bezanilla et al., 2000). Myo2p is "conventional" in the sense that two heavy chains form a rodshaped, coiled-coil tail that is insoluble at low ionic strength, presumably owing to the formation of filaments (Bezanilla and Pollard, 2000; Lord and Pollard, 2004). Myo2p is the second known contractile ring protein to concentrate around the equator of dividing cells, independent of actin filaments and septins, which arrive later (Naqvi et al., 1999; Wu et al., 2003). Accumulation of Myo2p at the cell equator is dependent on dephosphorylation of a phosphoserine at the C terminus of its tail (Motegi et al., 2004). Compaction of Myo2p into a tight contractile ring depends on actin filaments (Motegi et al., 2000; Wu et al., 2003) and Rng3p (Wong et al., 2000; Lord and Pollard, 2004). Myp2p is "unconventional," because the tail has a large insert between two segments of heptad repeats. This allows isolated tail polypeptides to fold back to form an antiparallel coiled-coil, indicating that Myp2p is monomeric with a single head (Bezanilla and Pollard, 2000). In spite of this unconventional structure, the tails are insoluble at physiological ionic strength. Myp2p joins the contractile ring $25 \mathrm{~min}$ after Myo2p (Wu et al., 2003) whereupon it acts to stabilize the contractile ring (Mulvihill and Hyams, 2003).

Budding yeast (Saccharomyces cerevisiae) myosin-II, Myo1p, contributes to cytokinesis and primary septum formation (Watts et al., 1987; Bi et al., 1998; Lippincott and Li, 1998; Schmidt et al., 2002). In certain strain backgrounds (e.g., BF264-Du), myo1s cells are viable despite morphological defects at the division site and failure of cytokinesis in some cells (Bi et al., 1998). In other backgrounds (e.g., W303a), the severity of cytokinesis defects results in lethality (Tolliday et al., 2003). Mlc1p is the ELC and Mlc2p is the RLC for Myo1p (Luo et al., 2004). Like the Myp2p tail, the Myo1p 
Table 1. Yeast strains employed in this study

\begin{tabular}{|c|c|c|}
\hline Strain & Genotype & Source \\
\hline \multicolumn{3}{|c|}{ Budding yeast } \\
\hline JC 19 & $M A T \alpha$ sst2 & J. Chant \\
\hline JC 1284 & 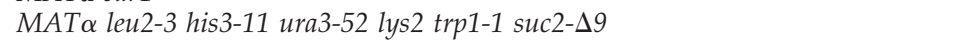 & J. Chant \\
\hline JC 1401 & 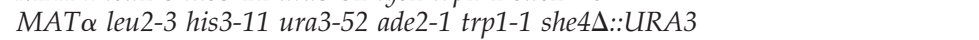 & J. Chant \\
\hline JC 1609 & $\begin{array}{l}\text { MATa leu2-3 his3-11 ura3-52 ade2-1 trp1-1 suc2- } 9 \text { myo1s::HIS3 (BF264-Du } \\
\text { background) }\end{array}$ & J. Chant \\
\hline \multicolumn{3}{|c|}{ 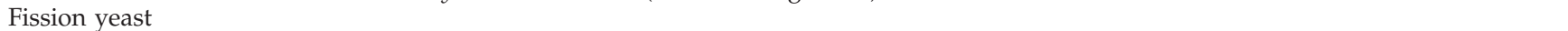 } \\
\hline FY 527 & $h^{-}$leu1-32 his3-D1 ura4-D18 ade6-M216 & S. Forsburg \\
\hline TP 5 & $h^{-}$leu1-32 his7-366 ura4-D18 ade6-M210 myp2s::his $7^{+}$ & Bezanilla et al. (1997) \\
\hline TP 73 & $h^{-}$leu1-32 his7-366 ura4D18 ade6-M216 myo2-E1 & M. Balasubramanian \\
\hline JW 994 & $h^{-}$leu1-32 his3-D1 ura4-D18 ade6-M216 kan ${ }^{R}: G F P-m y p 2$ & Wu et al. (2003) \\
\hline EL 101 & $h^{-}$leu1-32 his3-D1 ura4-D18 ade6-M216 kan ${ }^{R}: G F P-\Delta$ bp1-2547 myp2 & This study \\
\hline
\end{tabular}

tail is relatively long, with two distinct coiled-coil regions separated by a segment unlikely to form a coiled-coil (Bezanilla and Pollard, 2000). No information is available on the physical properties of the Myo1p tail. Concentration of Myo1p in the contractile ring early in the cell cycle at $\mathrm{G}_{1} / \mathrm{S}$ phase depends on the septin protein complex but not on actin filaments (Bi et al., 1998; Lippincott and Li, 1998).

We investigated the functional relationships among Myo1p, Myo2p, and Myp2p by using complementation and localization experiments. Remarkably, the Myo1p tail alone fully supports localization to the contractile ring and cytokinesis of both the BF264-Du and W303a strains of budding yeast. In contrast, both the heads and tails of Myo2p and Myp2p are necessary for their function in fission yeast. Our work reports the first known case of a myosin that maintains its cellular function without a motor domain and highlights the importance of myosin-II tails in cytokinesis.

\section{MATERIALS AND METHODS}

\section{Yeast Strains, Growth Conditions, Cell, and Genetic Methods}

Table 1 lists budding and fission yeast strains. Standard budding and fission yeast growth conditions, genetic methods, and DNA staining procedures

Table 2. Plasmids employed in this study

\begin{tabular}{|c|c|c|}
\hline Plasmid & Comment & Source \\
\hline pFA6a-kanMX6 & & Bahler et al. (1998) \\
\hline pFA6a-myp2 promoter-GFP-kanMX6 & & Wu et al. (2003) \\
\hline pFA6a-natMX4 & & This study \\
\hline pAG36 & natMX4 in pRS316 derivative & Goldstein and McCusker (1999) \\
\hline \multicolumn{3}{|l|}{ Budding yeast plasmids } \\
\hline YCp50-MYO1 & MYO1 clone & J. Chant \\
\hline$p R S-314$ & TRP1, low copy, centromeric vector & Sikorski and Hieter (1989) \\
\hline pPGT & MYO1 promoter-GFP-MYO1 terminator in $p R S 314$ & This study \\
\hline pGFP-MYO1 & pPGT encoding modular full-length Myolp & This study \\
\hline$p G F P-m y o 2$ & pPGT encoding full-length Myo2p & This study \\
\hline$p G F P-m y p 2$ & pPGT encoding full-length Myp2p & This study \\
\hline pGFP-MYO1H-myo2T & pPGT encoding Myo1p head-Myo2p tail chimera & This study \\
\hline pGFP-MYO1H-myp $2 T$ & $p P G T$ encoding Myo1p head-Myp2p tail chimera & This study \\
\hline pGFP-myo2H-MYO1T & pPGT encoding Myo2p head-Myolp tail chimera & This study \\
\hline pGFP-myp $2 H-M Y O 1 T$ & $p P G T$ encoding Myp2p head-Myo1p tail chimera & This study \\
\hline pGFP-MYO1T & pPGT encoding Myolp tail alone (aa 842-1928) & This study \\
\hline \multicolumn{3}{|l|}{ Fission yeast plasmids } \\
\hline$p 572-81 X$ & ura4, 81nmt1 promoter-C-terminal GFP vector & S. Forsburg \\
\hline p573-81X & LEU2, 81nmt1 promoter-N-terminal GFP vector & S. Forsburg \\
\hline p81-myo2 & p573-81X encoding full-length Myo2p & Bezanilla and Pollard (2000) \\
\hline p81-myp2 & p573-81X encoding full-length Myp2p & Bezanilla and Pollard (2000) \\
\hline p81-myo2H & p572-81X encoding Myo2p head alone (aa 1-815) & This study \\
\hline p81-mуо $2 \mathrm{H}-$ тур $2 \mathrm{~T}$ & $p 573-81 X$ encoding Myo2p head-Myp2p tail chimera & Bezanilla and Pollard (2000) \\
\hline p81-myp $2 \mathrm{H}-m y o 2 \mathrm{~T}$ & p573-81X encoding Myp2p head-Myo2p tail chimera & Bezanilla and Pollard (2000) \\
\hline p81-MYO1 & p573-81X encoding full-length Myo1p & This study \\
\hline p81-туо2Н-MYO1T & p573-81X encoding Myo2p head-Myo1p tail chimera & This study \\
\hline p81-MYO1H-myo2T & p573-81X encoding Myo1p head-Myo2p tail chimera & This study \\
\hline p81-mчр $2 H-M Y O 1 T$ & p573-81X encoding Myp2p head-Myo1p tail chimera & This study \\
\hline p81-MYO1H-myp2T & p573-81X encoding Myolp head-Myp2p tail chimera & This study \\
\hline
\end{tabular}


were used (Rose et al., 1990; Moreno et al., 1991). We used two different myo1 strains with different genetic backgrounds: JC 1609 in the BF264-Du background, in which deletion of MYO1 is not lethal; and RLY 1236 in the W303a background, in which MYO1 is essential (Tolliday et al., 2003). We used the JC1609 strain for complementation experiments using haploids. Derivatives of JC 1609 (referred to as JC 1609 for simplicity) were used after thorough backcrossing with a congenic wild-type strain to eliminate the emergence of suppressors, which are known to arise in this myo1s haploid background (Tolliday et al., 2003). To test the ability of constructs to rescue in a background where $M Y O 1$ represents an essential gene, we transformed a heterozygote diploid (RLY 1236 myo1s:kan ${ }^{R} / M Y O 1$ ) with appropriate plasmids. Transformants were sporulated and tetrads were dissected onto YPDa plates. Segregants were further tested for geneticin resistance $\left(k a n^{R}\right)$ on YPDa plates containing $200 \mathrm{mg} / \mathrm{ml}$ geneticin (Invitrogen, Carlsbad, CA), plasmid possession on CSM-Trp ${ }^{-}$plates, and mating type by halo assays using a- (JC 19) and $\alpha$-factor (JC 65) tester strains. Budding yeast strains carrying green fluorescent protein (GFP) fusion protein plasmids were used in complementation and localization experiments after growing exponentially in CSM-Trp ${ }^{-}$medium.

Fission yeast strains carrying GFP fusion protein plasmids were selected on EMM minus leucine plates containing $5 \mu \mathrm{g} / \mathrm{ml}$ thiamine. Cells were used in experiments after growing exponentially in EMM minus leucine plus $5 \mu \mathrm{g} / \mathrm{ml}$ thiamine (repressing conditions for complementation experiments) or plus 5-50 ng/ml thiamine (semirepressing conditions for localization experiments).

Truncation and GFP tagging of chromosomal myp2 was achieved using the one-step PCR and homologous integration method of Bahler et al. (1998). The geneticin-resistance gene $\left(\mathrm{kan}^{R}\right)$ and nourseothricin-resistance gene $\left(n a t^{R}\right)$ were used to truncate myp 2 at the $\mathrm{N}$ terminus ("headless") and $\mathrm{C}$ terminus ("tailless"), respectively. The N-terminal truncation resulted in replacement of the first 849 amino acids of Myp2p with GFP, whereas the C-terminal truncation resulted in removal of the last 1255 amino acids. Oligonucleotides containing $\mathrm{kan}^{R}$ or $\mathrm{nat}^{R}$ were integrated into chosen strains through high efficiency transformation. Integrants were subsequently selected on YE5S plates containing $100 \mu \mathrm{g} / \mathrm{ml}$ geneticin or $50 \mu \mathrm{g} / \mathrm{ml}$ clonNAT (Werner Bioreagents, Hamburg, Germany). Oligonucleotides were amplified with a High Fidelity polymerase (Roche Diagnostics, Indianapolis, IN) from $p F A 6 a-m y p 2$ promoter-GFP-kanMX6 or $p F A 6 a-n a t M X 4$ accordingly. Correct integration was confirmed by diagnostic PCR of genomic DNA using oligonucleotides derived from the sequence of selective markers and the target site. The phenotype and localization of integrated Myp2p GFP fusion proteins were analyzed after exponential growth in EMM medium $\left(20^{\circ} \mathrm{C}\right)$ and YE5S medium $\left(30^{\circ} \mathrm{C}\right)$, respectively.

A

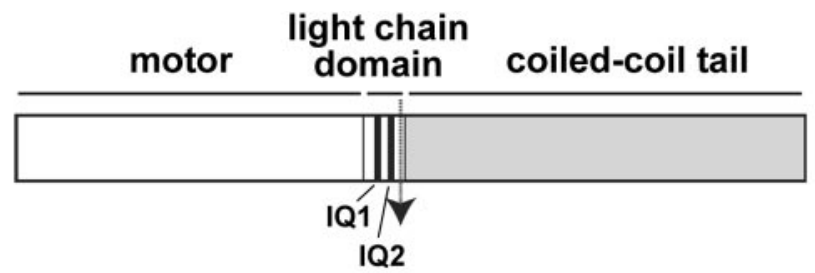

Figure 1. The Myo1p tail alone provides the full function of Myo1p during cytokinesis in the BF264-Du strain background. (A) Diagram of the myosin-II domain structure shown for a hypothetical myosin-II sequence. The N-terminal catalytic domain or head binds ATP and actin filaments. The light chain domain binds the ELC (IQ1) and RLC (IQ2). The C-terminal tail forms a dimer that assembles into bipolar filaments. The arrow indicates the site at the beginning of the tail where an NcoI site was engineered into the coding sequence of $M Y O 1$, myo2, and myp 2 to facilitate construction of plasmids containing chimeras and the headless Myo1Tp (lacking both the catalytic and light chain domains). (B) Differential interference contrast (DIC) (left) and fluorescence (right) micrographs of budding yeast myo1s cells expressing GFPMyo1p ( $p$ GFP-MYO1), GFP ( $p P G T)$, GFPMyo2H-Myo1Tp ( $p$ GFP-myo2H-MYO1T), or GFP-Myp2H-Myo1Tp ( GFP-myp2H-MYO1T). Cells were grown and mounted in CSM-Trp medium. (C) In a separate experiment budding yeast cells (as in B and F) were grown in CSM-Trp ${ }^{-}$medium and scored for cytokinesis defects measured as the fraction of cells forming connected cell chains, as described by Tolliday et al. (2003). (D) DIC (left) and fluorescence (right) micrographs of budding yeast myo1s she4s cells expressing GFPMyo1p ( $p$ GFP-MYO1), GFP ( $p P G T)$, or GFPMyo2H-Myo1Tp ( $p$ GFP-myo2H-MYO1T). (E) In a separate experiment, budding yeast cells (as in D) were grown in CSM-Trp ${ }^{-}$medium and scored for cytokinesis defects. (F) DIC (left) and fluorescence (right) micrographs of budding yeast myo1s cells expressing GFPMyo1Tp (Myo1p tail). Bars, $5 \mu \mathrm{m}$.

B
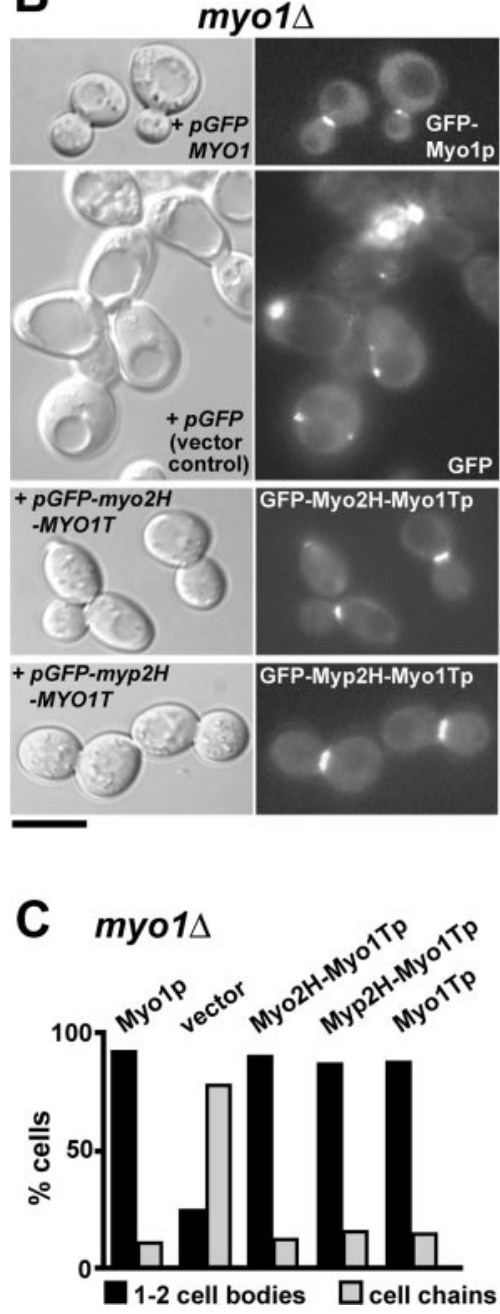

D myo1 $\Delta$ she4 $\Delta$

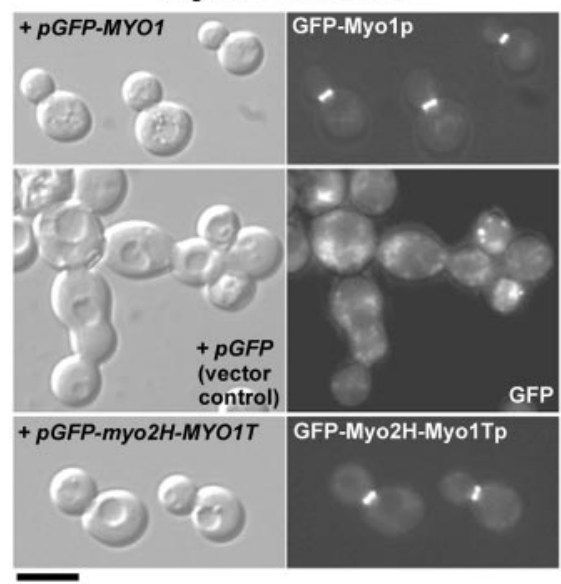

E myo1 she4 $\Delta$

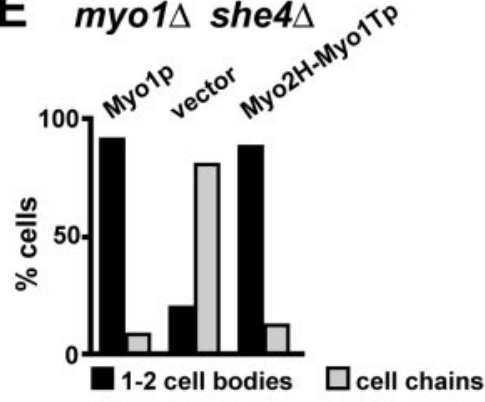

$\mathbf{F}$

myo1s

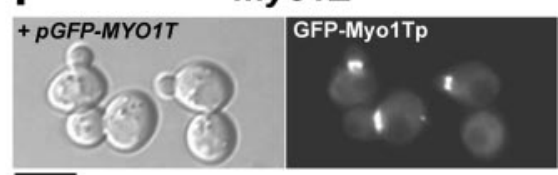


GFP fusion proteins/stains were imaged in cells as described previously (Lord and Pollard, 2004). Images were captured with an Olympus IX71 microscope by epifluorescence illumination with a PlanApo 60× (1.4 numerical aperture) objective and recorded with an Orca-ER cooled charge-coupled device camera (Hamamatsu, Bridgewater, NJ). For spinning disk confocal microscopy, the microscope was connected to a confocal scanner (model UltraView RS; PerkinElmer Life and Analytical Sciences, Boston, MA), and the images were captured with a PlanApo $100 \times$ (1.4 numerical aperture) objective. Images were acquired and processed using MetaMorph, Utra View RS, Image J, and PhotoShop software.

\section{Plasmid Construction}

Table 2 lists the plasmid constructs used in this study. The fidelity of constructs was verified by automated DNA sequencing (Keck Facility, Yale University, New Haven, CT).

pPGT. An 800-base pair region upstream of the MYO1 open reading frame (ORF) (encompassing the MYO1 promoter) was amplified from budding yeast genomic DNA with $5^{\prime}$ KpnI-prom (GGTACCGCATAGACGATCTCTACGAC) and 3' Xhol-prom (CTCGAGTATTGCTGTTGTTGTCCTGTC). The DNA was ligated into KpnI/XhoI linearized pRS314. A XhoI/NotI GFP fragment was liberated from $p 573-81 \mathrm{X}$ and ligated into XhoI/NotI linearized pRS314-MYO1promoter plasmid. A 1-kb terminator region immediately downstream of the MYO1 ORF was amplified from genomic DNA with $5^{\prime}$ NotISall-term (GCGGCCGCTTTAGTCGACGACGACACGAGCGTTATATAC) and 3' SacI-term (GAGCTCGAATTATTCCACATCCAGATCGG) and ligated into NotI/SacI linearized pRS314-MYO1promoter-GFP to yield pPGT.

pGFP-myo2/-myp2. NotI/SalI myo2 and myp2 fragments were liberated from p573-81X-myo2 and p573-81X-myp2, respectively, and ligated into NotI/SalI linearized $p P G T$, generating $p G F P-m y o 2$ and $p G F P-m y p 2$.

pGFP-MYO1/MYO1H-myo2T/-MYO1H-myp2T/-myo2H-MYO1T/-myp2HMYO1T. We constructed chimeric myosin head-tail budding yeast constructs using a cloning strategy similar to that used to generate fission yeast myosin-II chimeras (Bezanilla and Pollard, 2000). As with myo2 and myp2, the conserved sequence CCTTGG (encoding amino acids ProTrp in each myosinII) was found at the junction of the DNA regions encoding the head and tail of Myolp (base pairs 2524-2529, aa 842-843). When constructing the $3^{\prime}$ head and $5^{\prime}$ tail primers for $M Y O 1$, we took advantage of this sequence by making a silent point mutation (CCTTGG to CCATGG) to engineer an NcoI site (Figure 1A). The MYO1 head and tail encoding fragments were amplified from YCp50-MYO1 using the following primers: 5' NotI-MYO1 (GCGGCCG CATGACCGGCGGGCAGTCTTGC) and $3^{\prime}$ SalI-NcoI-MYO1 head (ACGCGTCGACGTCAAACCATGGATCTTCCTTCACCAGTC); $5^{\prime} \mathrm{NcoI}$ MYO1 tail (GACTGGTGAAGGAAGATCCATGGTTTA) and 3' Sall-MYO1 (GTCGACCGTTAACTGAAAATTTTACTCTGTGC). Two naturally occurring NcoI sites are present within the GFP-MYO1 head sequence (one in the GFP ORF and one in the MYO1 head sequence). To overcome this complication, we used the following cloning strategy: $p P G T$ was linearized with NcoI and SalI, and an NcoI/SalI MYO1 tail fragment was subsequently inserted to yield a construct carrying a 5' portion of GFP fused to the MYO1 tail sequence. In parallel, a NotI/SalI-NcoI MYO1 head fragment was inserted into NotI/SalI linearized $p P G T$. This construct was in turn subjected to partial NcoI digestion to liberate a $3^{\prime}$ portion of GFP fused to the complete MYO1 head sequence (i.e., with its naturally occurring, internal $\mathrm{NcoI}$ site intact). This fragment (" $3 \mathrm{~kb}$ MYO1 head") was subsequently inserted into the plasmid carrying part of GFP fused to the MYO1 tail (see above) via the NcoI site to yield $p$ GFP-MYO1. Correct orientation of the $3 \mathrm{~kb}$ MYO1 head fragment was tested using restriction digest analysis. The modular head-tail GFP-MYO1 construct was fully functional as judged by its ability to complement myo1s strains. Having generated a modular head-tail construct compatible (in terms of reading frame, and the presence of an NcoI site at the head-tail junction) with myo2 and myp2 chimeric modular constructs (p81myo2H-myp2T and p81-myp2Hmyo2T), chimera constructs ( $p$ GFP-MYO1H-myo2T/-MYO1H-myp2T/-myo2HMYO1T/-myp2H-MYO1T) were generated by replacing MYO1 head and tail modules using appropriate NcoI GFP-head and NcoI/SalI tail fragments (as detailed above for $p G F P-M Y O 1$ ). Regular digests (not partial digests) were used to liberate GFP-myo2/myp2 3kb head fragments because, unlike MYO1, neither myo2 or myp 2 possesses naturally occurring NcoI sites.

pGFP-MYO1T. The GFP-Myo1p tail fusion was constructed by replacing the NcoI GFP-MYO1 head fragment (3kb MYO1 head) in $p G F P-M Y O 1 H-M Y O 1 T$ with an NcoI GFP fragment generated from a GFP sequence amplified from p573-81X with primers: $5^{\prime}$ XhoI-GFP (CTCGAGATGTCTTTGAGTAAAGGAGAAGAACTTTTCAC) and $3^{\prime} \mathrm{NcoI}$-GFP (CCATGGTTTGTATAGTTCATCCATGCCATG). Correct orientation of GFP was tested using restriction digests.

p81-MYO1H-myo2T/-MYO1H-myp2T/-myo2H-MYO1T/-myp2H-MYO1T. Fission yeast MYO1-myo2/myp2 chimera plasmids were constructed using head-tail module replacement (as described for budding yeast $p P G T$ chimera plasmids; see above). p81-myo2H-myp2T and p81-myp2H-myo2T plasmids were linearized appropriately and used to generate the various chimera constructs by religation with appropriate modules.

Table 3. Functionality of myosin-II chimeras and truncations

Tail

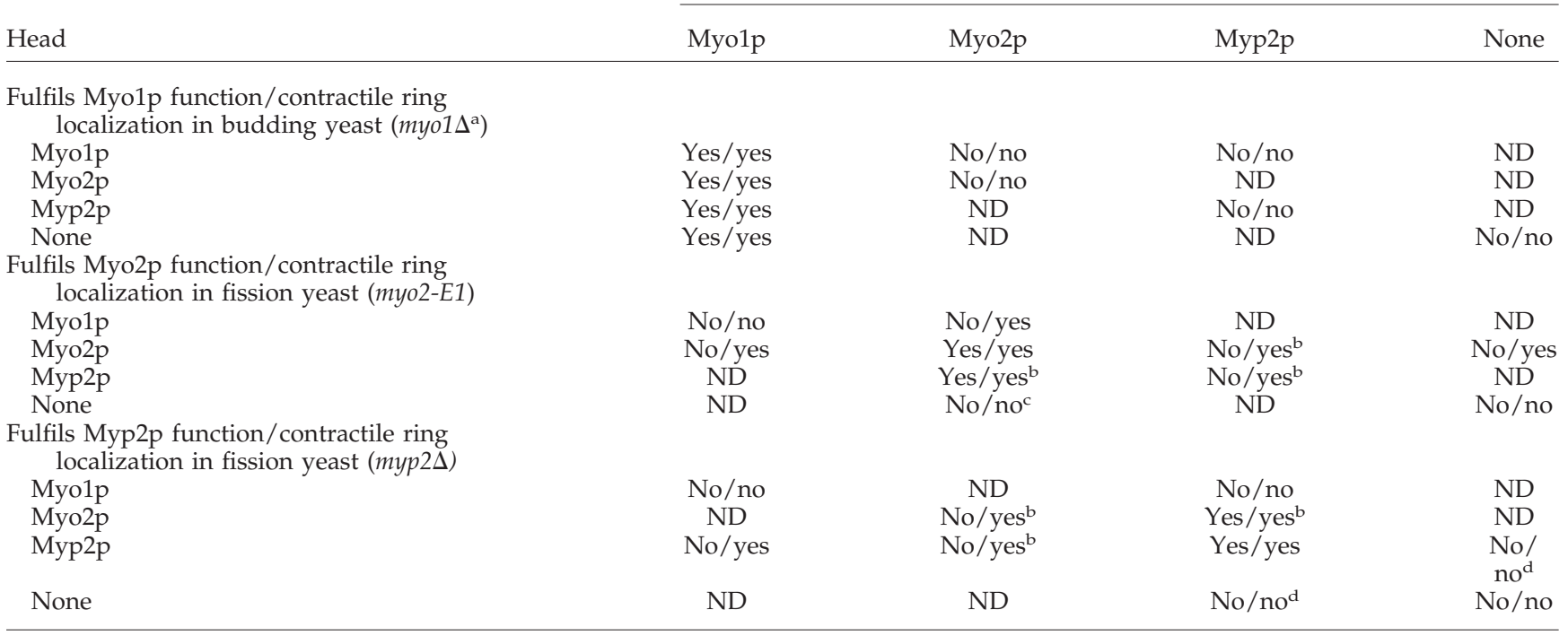

ND, no data.

a myo1s in BF264-Du background (JC 1609).

b Bezanilla and Pollard, 2000.

c Naqvi et al. (1999).

d Data from GFP-fusions constructed by chromosomal integration of GFP into fission yeast (FY 527). 
A
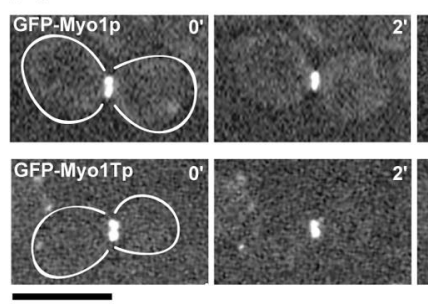
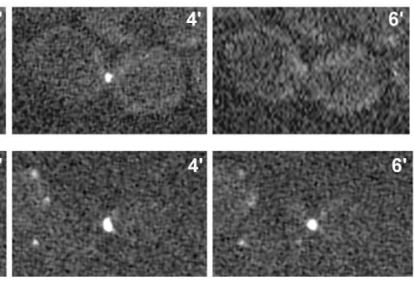

B

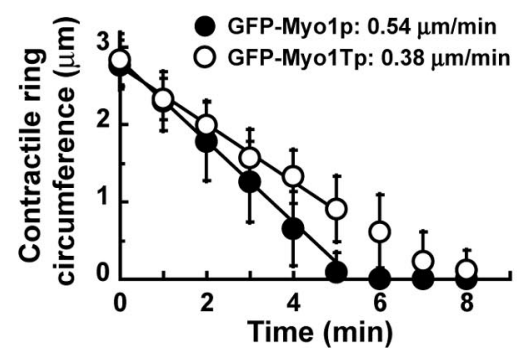

C

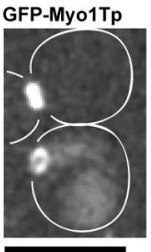

Figure 2. Dynamics of the Myo1p tail contractile rings are similar to those of full-length Myo1p contractile rings. (A) Time-lapse spinning disk confocal fluorescence micrographs of myo1s strain (BF264-Du) cells expressing GFP-Myo1p (top) or GFP-Myo1Tp (bottom) during contractile ring constriction. Stacks of six confocal z sections at intervals of $0.5 \mu \mathrm{m}$ were collected (projected at maximum intensity) at each time point. Dividing cells are outlined in white in the first panels. (B) Plot of average contractile ring circumference versus time for 20 GFP-Myo1p cells (closed circles) and 20 GFP-Myo1Tp cells (open circles). Time zero represents the point at which rings initiate contraction. The slopes of these plots are the rates of ring constriction. (C) Confocal fluorescence micrograph of two cells carrying the GFP-Myo1Tp construct (as described in A). The lower cell is orientated such that a complete, uninterrupted contractile ring is apparent. Bars, $5 \mu \mathrm{m}$.

p81-MYO1. A NotI/SalI MYO1 fragment was liberated from $p G F P-M Y O 1$ and ligated into NotI/SalI linearized p573-81X.

p81-myo2H. A subfragment-1 (S1)-like Myo2Hp-GFP ("S1-like") head fusion was constructed by inserting a myo2 head fragment into $p 572-81 X$. The head (base pairs 1-2445; aa 1-815) was amplified using the primers 5' NotI-myo2-S1 (GCGGCCGCGGCGGTGGAATGACAGAAGTAATATCTAATAAAATAACTGC) and 3' NotI-myo2-S1 (GCGGCCGCCGGGCCTTAGATTGAAAAATAACTTAGC).

pFA6a-natMX4. This plasmid was generated by replacing the NcoI/SacI kan fragment from $p F A 6 a-k a n M X 6$ (Bahler et al., 1998) with an NcoI/SacI nat ${ }^{R}$ fragment derived from pAG36 (Goldstein and McCusker, 1999).

\section{Database Searches and Sequence Analysis}

Uncharacterized fungal myosin-II amino acid sequences were identified from various genomes via sequence homology comparisons with the $S$. pombe Myo2p motor. The following resources were used: Aspergillus fumigatus Genome Project (http://www.tigr.org/tdb/e2k1/afu1/), Munich Information Center for Protein Sequences (http://mips.gsf.de/projects/fungi/neurospora.-html), Candida albicans Research Lab (http://candida.bri.nrc.ca/candida/ index.cfm), and Cryptococcus neoformans Genome Project (http://www. tigr.org/tdb/e2k1/cna1/). The probability of coiled-coil conformation within myosin-II tail sequences was calculated by the COILS program (Lupas et al., 1991; http:/ / www.ch.embnet.org/software/COILS_form.html) using a window width of 28 amino acids.

\section{RESULTS}

Budding Yeast Myo1p Functions without Its Head

Initial studies using myosin-II chimeras (consisting of various combinations of Myo1p, Myo2p, and Myp2p heads and tails) led to a surprising observation. Like full-length GFPMyo1p, chimeras consisting of either the GFP-Myo2p head or the GFP-Myp2p head fused to the Myo1p tail localized to contractile rings and rescued cytokinesis defects of the budding yeast myo1s strain (Figure 1B and Table 3). Like GFPMyo1p (Figure 1B), expression of either GFP-Myo2HMyo1Tp or GFP-Myp2H-Myo1Tp fully overcame the undivided, multinucleate phenotype of myo1s cells (Figure 1C). The finding was surprising, because Myo2p motor function and fission yeast cytokinesis require the UCS protein Rng3p (Wong et al., 2000; Lord and Pollard, 2004). Naturally, Rng3p is not present in budding yeast, and She4p, the only UCS protein common to budding yeast, is specific for type I and V myosins, not type II Myo1p (Toi et al., 2003; Wesche et al., 2003). To rule out the possibility that She4p was substituting for Rng3p in budding yeast to promote the motor activity of the GFP-Myo2H-Myo1Tp, we tested the ability of this chimera to rescue loss of Myo1p function in a double deletion mutant lacking both MYO1 and SHE4. In the absence of any UCS protein, GFP-Myo2H-Myo1Tp still fully supported Myo1p function (Figure 1, D and E).

To test whether the motor domain is required for myosin-II function in budding yeast, we constructed a truncated form of Myolp consisting of GFP fused to the Myo1p tail. Remarkably, plasmid expression of this headless Myo1p construct (GFP-Myo1Tp) fully restores Myo1p function in a myo1s strain (BF264-Du) and concentrates in a ring at the

Table 4. Summary of complementation results for haploid segregants generated in the W303a background after plasmid transformation, sporulation, and tetrad dissection of a myo1 $/$ MYO1 heterozygous diploid (RLY 1236)

\begin{tabular}{lcrr}
\hline & \multicolumn{2}{c}{ Recovered segregants } \\
\cline { 2 - 4 } Plasmid & TRP1 $^{+\mathrm{a}}$ & $k^{R} \mathrm{~b}$ & TRP1 $^{+} \mathrm{kan}^{R \mathrm{c}}$ \\
\hline pPGT (vector alone) & 32 & 0 & 0 \\
pGFP-MYO1 & 62 & 29 & 29 \\
pGFP-MYO1-Tail & 55 & 23 & 23 \\
\hline
\end{tabular}

${ }^{a} T R P 1^{+}$represents haploids carrying the indicated TRP1-containing plasmid from segregants generated from 25 tetrads (per transformant). Recovered segregants lacking a $T R P 1^{+}$phenotype have presumably undergone plasmid loss during sporulation and tetrad dissection onto YPDa plates.

${ }^{\mathrm{b}} \mathrm{kan}^{R}$ represents haploid segregants that possess the $m y \mathrm{o} 1 \Delta: \mathrm{kan}^{R}$ deletion. Haploids lacking $k \mathrm{n}^{R}$ resistance (geneticin-sensitive) constitute segregants possessing an intact MYO1 gene.

${ }^{c}$ All $\mathrm{kan}^{R}$ segregants analyzed were found to be complemented $\left(T R P 1^{+}\right)$by either full-length GFP-Myo1p or the GFP-Myo1p tail. No kan ${ }^{R}$ segregants were derived from $p P G T$ (vector alone) transformants. These data are consistent with both GFP-Myo1p and the GFP-Myo1p tail supporting the essential function of Myo1p in this strain background (W303a) where myo1s is lethal. 
bud neck (Figures 1F and 2C). Like GFP-Myo1p, expression of GFP-Myo1Tp fully overcame the undivided, multinucleate phenotype of myo1s cells (Figure 1C).

The ring of GFP-Myo1Tp in the bud neck constricted during cytokinesis (Figure 2A) at 70\% the rate of myo1s cells expressing full-length Myo1p (Figure 2B). This small reduction in the rate of contraction had no effect on the ability of GFP-Myo1Tp to fully complement a myo1s strain (Figure 1, $\mathrm{B}$ and $\mathrm{C})$, because the cell doubling time was indistinguishable from that of myo1s cells carrying GFP-Myo1p (our unpublished data). The slightly reduced rate of Myo1Tp contraction may reflect a nonessential role for the Myolp motor in contractile ring disassembly, as proposed for Mlc $2 p$ (Luo et al., 2004). To confirm that the Myo1p tail contractile rings, such as those shown in Figure 2A, were actually true rings, we imaged cells orientated in the mounting medium with their emerging buds pointing toward the objective. Like full-length Myo1p (Bi et al., 1998), the Myo1p tail formed complete, uninterrupted rings (Figure 2C). Thus, the budding yeast contractile ring constricts competently during cytokinesis in the absence of myosin-II motor activity.

Myo1p tail alone was also sufficient for function in the W303a strain, where loss of MYO1 is lethal. We transformed the heterozygote myo1 $/$ MYO1 diploid with plasmids carrying GFP (vector alone), GFP-Myo1p, or GFP-Myo1Tp. Transformants were sporulated and tetrads dissected to test which of these plasmids rescue the lethality of haploid myo1s segregants. The vector alone did not rescue, whereas both GFP-Myo1p and GFP-Myo1Tp constructs rescued myo1s lethality as demonstrated by recovery of $\mathrm{kan}^{R} \mathrm{seg}$ regants complemented $\left(\mathrm{Trp}^{+}\right)$by $p P G T-M Y O 1$ or $p P G T$ MYO1T (Table 4). Both GFP-Myo1p and GFP-Myo1Tp concentrated in contractile rings at mother-bud necks (Figure 3 , $\mathrm{A}$ and $\mathrm{B})$. The phenotypes of strains rescued by full-length GFP-Myo1p and GFP-Myo1Tp were phenotypically identical (Figure 3, A-C).

\section{Fission Yeast Myosin-II Function Requires Both Heads and Tails}

In contrast to budding yeast Myolp, both the head and tail of fission yeast Myp2p are essential for function. Unlike full-length GFP-Myp2p, tailless (GFP-Myp2Hp) or headless (GFP-Myp2Tp) forms of Myp2p did not support myp2 function (Figure 4, A and B, and Table 3). Cells expressing truncated forms of Myp2p from integrated GFP-myp2 head or GFP-myp2 tail gene fusions displayed cytokinesis defects similar to a myp $2 \Delta$ strain (Figure 4, A and B). Both Myp2p tailless and headless GFP fusions failed to concentrate in contractile rings (Figure 4C and Table 1C). GFP-Myp2Tp formed punctate aggregates, whereas GFP-Myp2Hp exhibited a diffuse cytoplasmic localization pattern, very much unlike the distribution of full-length GFP-Myp2p (Figure $4 \mathrm{C})$.

Previous work established that Myo2p function requires both its catalytic head and tail domains. Point mutations in either the Myo2p head (Naqvi et al., 1999) or tail (Mulvihill et al., 2001; Motegi et al., 2004) can compromise function. Interestingly, a Myo2p Head-GFP fusion localized at contractile rings in wild-type or myo2-E1 cells, despite being unable to substitute functionally for myo2 (Table 3 and Figure 4D). Presumably, the Myo2p motor alone has a higher affinity for contractile ring actin filaments than the Myp2p motor domain. Expression of the Myo2p tail in myo2s strains fails to support contractile ring formation and cell division (Naqvi et al., 1999; Table 3) in spite of diffuse accumulation of the Myo2p tail at the medial division site.
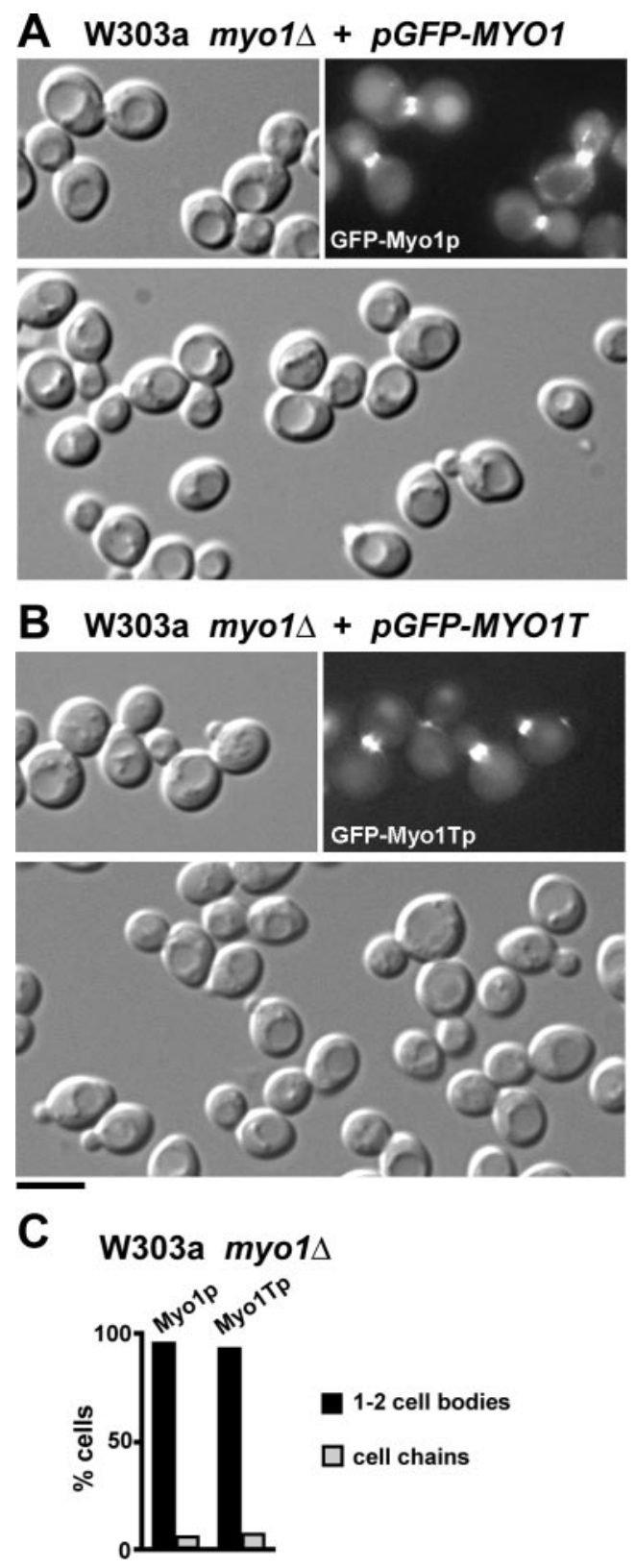

Figure 3. The Myo1p tail alone provides the full function of Myo1p during cytokinesis and cell growth in the W303a background. DIC (top left and bottom) and fluorescence (top right) micrographs of W303a budding yeast myo1s haploids expressing GFP-Myo1p ( $p$ GFP-MYO1) (A) and GFP-Myo1Tp ( $p$ GFP-MYO1T) (B). (C) In a separate experiment budding yeast cells (as in A and B) were grown in CSM-Trp ${ }^{-}$medium and scored for cytokinesis defects by quantitating the percent of cells forming connected cell chains. Bar, $5 \mu \mathrm{m}$.

Table 3 summarizes complementation and localization experiments for myosin-II head or tail truncations and all possible combinations of Myo1p/Myo2p/Myp2p head-tail chimeras. Both the negative and positive results are consistent with previous work (Naqvi et al., 1999; Bezanilla and Pollard, 2000) and the interpretations made in our present study. We do not emphasize the negative results, because we cannot rule out the possibility that negative complemen- 


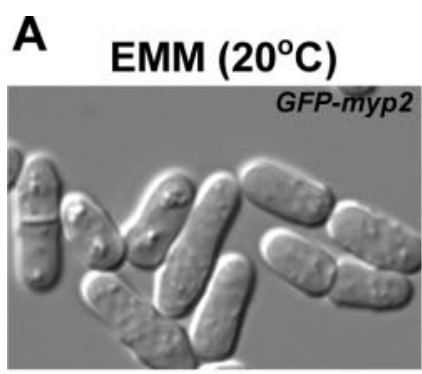

\section{B}
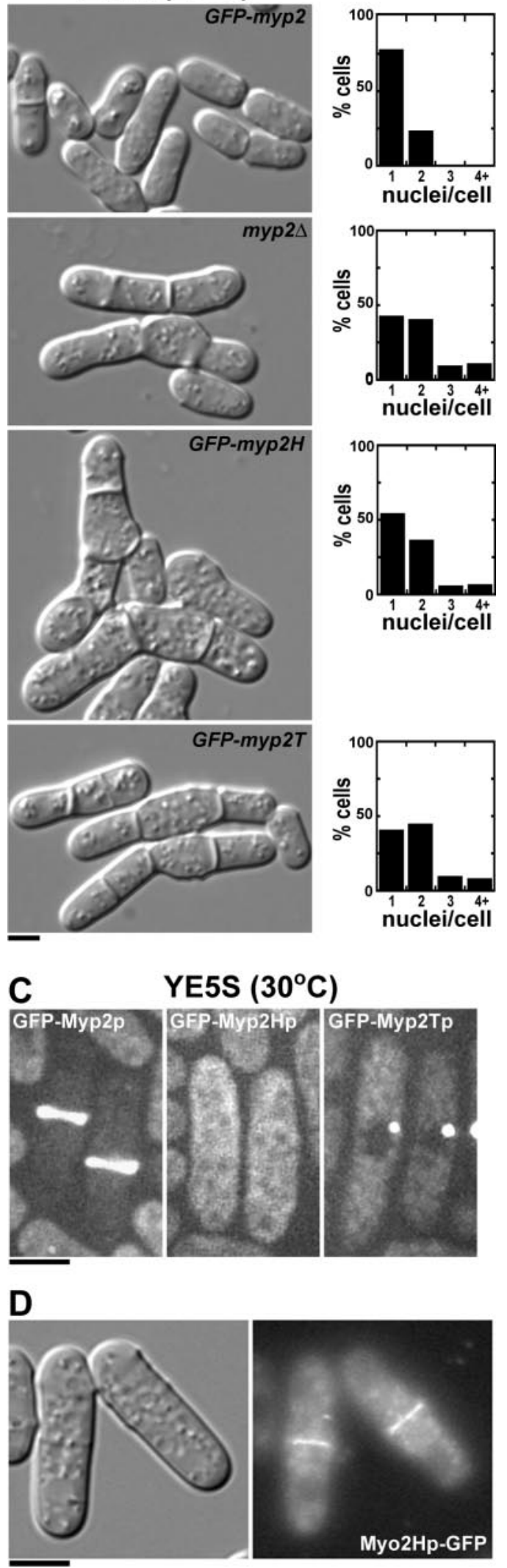

Figure 4. The head and tail of fission yeast Myp2p are required for its function. (A) DIC micrographs show the cell morphology for a fission yeast strain carrying a chromosomal GFP-myp2 fusion (JW tation/localization results arose from inefficient expression rather than failure to function.

\section{DISCUSSION}

In the contractile ring model of cytokinesis, actin filaments and myosin-II constrict the cleavage furrow. Presumably, the tails of the myosin-II molecules assemble bipolar filaments so that myosin heads can interact with oppositely polarized ring actin filaments in a sliding filament mechanism similar to muscle contraction. However, this and other recent work suggest that the role of myosin-II tails in cytokinesis is more complex.

Remarkably, the tail of Myo1p is essential for cytokinesis in budding yeast, but the heads are dispensable. Both the heads and tails of myosin-II are required for cytokinesis in other cells, including fission yeast. Other myosins can fulfill some of their roles in cells with compromised motor activity, but Myo1p is the first example of a myosin that functions in the complete absence of its motor domain. Mutant forms of fission yeast Myo2 $\mathrm{p}$ possessing only $5 \%$ of the wild-type in vitro motility activity (measured by actin filament gliding assays) support normal cytokinesis in vivo (Lord and Pollard, 2004). Furthermore, mutant forms of the essential type-I myosin from Aspergillus with only 1\% of the wild-type actin-activated ATPase activity still support normal cell growth with only minor phenotypic defects (Liu et al., 2001). On the other hand, Dictyostelium myosin-II lacking its ELC binds actin filaments but has $<10 \%$ of wild-type actin-activated ATPase activity (Chen et al., 1995). Cells lacking ELC fail to complete cytokinesis, yet still move reasonably normally (Chen et al., 1995; Xu et al., 2001; Laevsky and Knecht, 2003).

Why should the requirements for myosin-II in cytokinesis differ in budding yeast? Two possibilities come to mind. First, contractile force may not be required for cytokinesis in budding yeast. Growth of the septum might conceivably fill in the narrow neck between mother and bud, with the ring of actin filaments growing smaller by disassembly. Alternatively, another myosin, such as $S c$ Myo2p, may interact with actin filaments to constrict the ring. Budding yeast Myo2p is an essential type- $\mathrm{V}$ myosin that localizes to polarized growth sites and the bud neck (Johnston et al., 1991; Brockerhoff et al., 1994; Lillie and Brown, 1994; Govindan et al., 1995). Vesicle targeting dependent on Sc Myo2p facilitates septum closure (Wagner et al., 2002), which may produce the force to drive ring constriction in the absence of myosin-II motor activity. A role for this type- $\mathrm{V}$ myosin in cytokinesis is supported by genetic studies revealing that defects of a $m y 01 \Delta$ and a myo2-66 mutation are additive when combined (Lillie and Brown, 1998).

Despite lacking a motor domain the Myo1p tail concentrated in the ring structure at the bud neck and constricted

994), a myp $2 \Delta$ strain (TP5), and strains with chromosomal GFP-myp2 head (EL 102) or GFP-myp2 tail (EL 101) fusions replacing the myp2 gene. Strains were grown under restrictive conditions for testing Myp2p function (EMM medium, $20^{\circ} \mathrm{C}$ ). (B) In a separate experiment, cells were stained with Hoescht dye and scored for the number of nuclei per cell. (C) Fluorescence micrographs of GFPMyp2p (JW 994, left), GFP-Myp2Hp (EL 102, center), and GFPMyp2Tp (EL 101, right). Cells are shown after growth under permissive conditions (YE5S, $30^{\circ} \mathrm{C}$ ). (D) DIC (left) and fluorescence (right) micrographs for a strain (FY 527) bearing $p 81$-myo2H encoding the Myo2Hp-GFP fusion. Cells are shown after growth in EMM $\mathrm{Ura}^{-}$medium. Bars, $5 \mu \mathrm{m}$. 
Figure 5. Coiled-coil predictions for different myosin-II tails. Predictions were calculated using the COILS program (Lupas et al., 1991). The tails of Homo sapiens nonmuscle myosin-II-B, S. pombe Myp2p, S. cerevisiae Myo1p, S. pombe Myo2p, and four uncharacterized fungal type-II myosins (from A. fumigatus, $N$. crassa, C. albicans, and C. neoformans) were compared. $x$-axis, amino acid position. $y$-axis, probability of forming a coiled-coil.
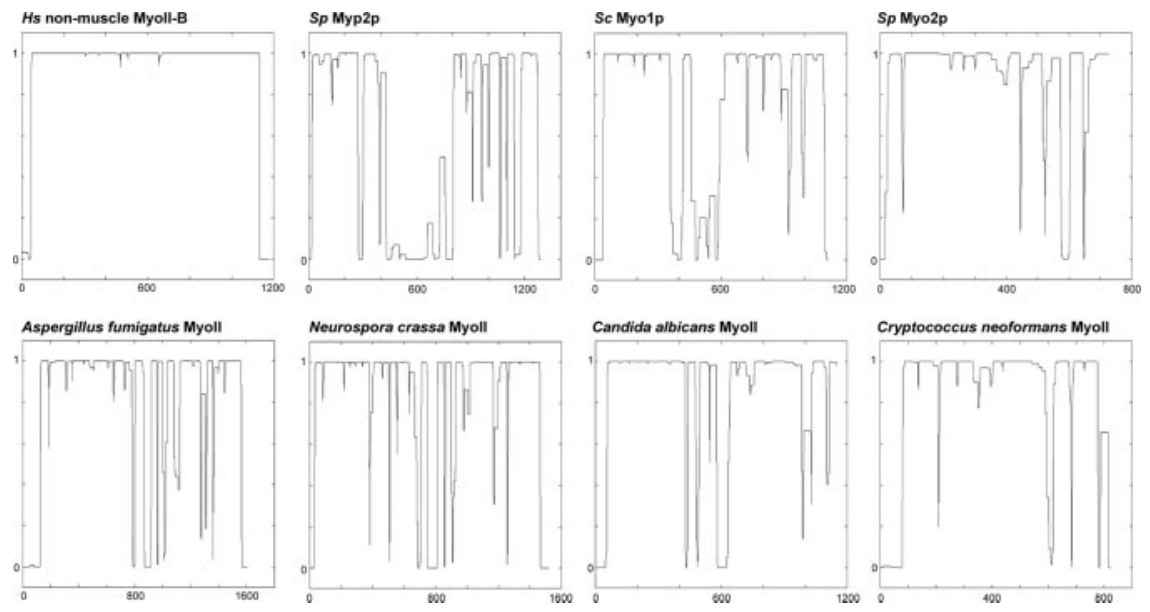

with almost wild-type dynamics. Tolliday et al. (2003) reported an unpublished observation that a construct consisting of the C-terminal 868 amino acids of Myo1p localizes to the bud neck. However, overexpression of this C-terminal region of Myo1p from the GAL1 promoter was toxic resulting in cells with cytokinesis defects reminiscent of a myo1s strain (Tolliday et al., 2003). This phenotype presumably stems from complications caused by an overabundance of the Myo1p tail, because we find that expression of the Myo1p tail from its native promoter had no negative effects on cytokinesis.

Because the function of budding yeast myosin-II does not depend on its motor domain, its tail must contribute productively to cytokinesis in some way. Myo1p tails might form filaments or interact with septins and/or other structural proteins to stabilize the bud neck during bud outgrowth, allowing other mechanisms such as new membrane addition and septum formation to close the narrow orifice. Indeed, ring contraction and primary septum formation are interdependent (Schimdt et al., 2002; VerPlank and Li, 2005), suggesting a role for Myo1p in coordinating septation and contractile ring closure. Myosin-II contributes to the mechanical stability of the cortex in Dictyostelium (Pasternak et al., 1989), although this is thought to depend on interaction of the heads with actin filaments.

Assembly of contractile rings also differs in the two yeast. Budding yeast Myolp occurs at the division site much earlier in the cell cycle $\left(G_{1} / S\right)$ than in other cells. Further Myo1p localization depends on assembly of the septin complex (Bi et al., 1998; Lippincott and Li, 1998). Fission yeast septins are not essential and occur at the division site later than Myo2p and Myp2p (Wu et al., 2003; An et al., 2004).

\section{Features of the Myo1p Tail That May Support a Novel Role for Myosin-II in Fungal Cytokinesis}

Available genome sequences show that fungi have two types of myosin-II. Some, including S. pombe Myo2p and Cryptococcus neoformans MyoII, have tails like conventional animal myosin-II, consisting almost entirely a coiled-coil. Most sequenced fungal myosin-II molecules, including $S$. pombe Myo1p and S. cerevisiae Myp2p, have tails with two distinct regions of predicted coiled-coil separated by a nonhelical insert of 200-270 amino acids (Bezanilla et al., 1997; Figure 5). Other examples include MyoII from Aspergillus fumigatus, MyoII from Candida albicans, and MyoII from Neurospora crassa. These inserts of 200 or more residues include $5-10 \%$ prolines, which are rarely found in the coiled-coil tails of conventional myosin-II. Hydrodynamic analysis established that the isolated Myo2p tail forms conventional dimers, whereas the Myp2p tail is monomeric, folding back on itself to form an antiparallel coiled-coil (Bezanilla and Pollard, 2000). Although Myo1p and Myp2p are not functional homologues, the ability of the Myo1p tail to support cytokinesis may arise from an antiparallel coiled-coil structure similar to Myp2p. Given this novel structure, approaches other than simple truncations will be required to determine how the tail of Myolp functions in cytokinesis without its heads.

\section{Fission Yeast Type-II Myosins Function as Actin- dependent Motors}

Unlike budding yeast, fission yeast type-II myosins act like other conventional myosins, requiring both their heads and tails for function. Point mutations in either the head or tail of Myo2p can attenuate its function (Balasubramanian et al., 1998; Naqvi et al., 1999; Wong et al., 2000; Motegi et al., 2004), whereas truncated forms of Myp2p lacking either the head or tail fail to support Myp2p function. Second, unlike Myo1p, both Myo2p and Myp2p require actin filaments to form a compact contractile ring at anaphase A (Naqvi et al., 1999; Wu et al., 2003). Third, Myo2p contractile ring localization requires Rng3p, a motor regulator (Wong et al., 2000; Lord and Pollard, 2004), whereas the Rng3p homolog (She4p) is dispensable for the localization and function of Myo1p (Toi et al., 2003; Wesche et al., 2003). Fourth, loss of the $S$. pombe RLC (Rlc1p) results in temperature-sensitive lethality (Le Goff et al., 2000; Naqvi et al., 2000), whereas loss of the budding yeast RLC (Mlc2p) has only very subtle effects on Myo1p localization and cytokinesis (Luo et al., 2004). As in cells possessing headless Myo1p, deletion of MLC2 yields Myo1p contractile rings that disappear slower than in wild-type strains, suggesting a nonessential role for Mlc2p and Myo1p motor activity in ring disassembly (Luo et al., 2004). Such subtle effects on contractile ring dynamics suggest that the Myo1p motor may play a secondary, nonessential role in budding yeast cytokinesis. The fact that Myo2p and Myp2p motor activity is essential for myosin-II function represents an advantage for fission yeast in studying cytokinesis powered by conventional myosin-II behavior.

\section{ACKNOWLEDGMENTS}

We thank John Chant for budding yeast strains and plasmids. We thank Jian-Qiu Wu for the myp2 promoter plasmid and the GFP-Myp2p strain, 
Mohan Balasubramanian for the myo2-E1 strain, Rong Li for the myo1 heterozygote W303a strain, and Susan Forsburg for numerous fission yeast strains and plasmids. We thank members of the Pollard laboratory for helpful discussions. This work was supported by National Institutes of Health Grant GM-26231 to T.D.P.

\section{REFERENCES}

An, H., Morrell, J. L., Jennings, J. L., Link, A. J., and Gould, K. L. (2004) Requirements of fission yeast septins for complex formation, localization, and function. Mol. Biol. Cell 15, 5551-5564.

Bahler, J., Wu, J.-Q., Longtine, M. S., Shah, N. G., McKenzie, 3rd, A., Steever, A. B., Wach, A., Philippsen, P., and Pringle, J. R. (1998). Heterologous modules for efficient and versatile PCR-based gene targeting in Schizosaccharomyces pombe. Yeast 14, 943-951.

Balasubramanian, M. K., McCollum, D. Chang, L., Wong, K. C., Naqvi, N. I., He, X., Sazer, S., and Gould, K. L. (1998). Isolation and characterization of new fission yeast cytokinesis mutants. Genetics 149, 1265-1275.

Bezanilla, M., Forsburg, S. L., and Pollard, T. D. (1997). Identification of a second myosin-II in Schizosaccharomyces pombe: Myp2p is conditionally required for cytokinesis. Mol. Biol. Cell 8, 2693-2705.

Bezanilla, M., and Pollard, T. D. (2000). Myosin-II tails confer unique functions in Schizosaccharomyces pombe: characterization of a novel myosin-II tail. Mol. Biol. Cell 11, 79-91.

Bezanilla, M., Wilson, J. M., and Pollard, T. D. (2000). Fission yeast myosin-II isoforms assemble into contractile rings at distinct times during mitosis. Curr. Biol. 10, 397-400.

Bi, E., Maddox, P., Lew, D. J., Salmon, E. D., McMillan, J. N., Yeh, E., and Pringle, J. R. (1998). Involvement of an actomyosin contractile ring in Saccharomyces cerevisiae cytokinesis. J. Cell Biol. 142, 1301-1312.

Bresnick, A. R. (1999). Molecular mechanisms of nonmuscle myosin-II regulation. Curr. Opin. Cell Biol. 11, 26-33.

Brockerhoff, S. E., Stevens, R. C., and Davis, T. N. (1994). The unconventional myosin, Myo2p, is a calmodulin target at sites of cell growth in Saccharomyces cerevisiae. J. Cell Biol. 124, 315-323.

Chen, T.-L.L., Kowalczyk, P. A., Ho, G., and Chisholm, R. L. (1995). Targeted disruption of the Dictyostelium myosin essential light chain gene produces cells defective in cytokinesis and morphogenesis. J. Cell Sci. 108, 3207-3218.

Echard, A., Hickson, G. R., Foley, E., and O'Farrell, P. H. (2004). Terminal cytokinesis events uncovered after an RNAi screen. Curr. Biol. 14, 1685-1693.

Goldstein, A. L., and McCusker, J. H. (1999). Three new dominant drug resistance cassettes for gene disruption in Saccharomyces cerevisiae. Yeast 15, 1541-1553.

Govindan, B., Bowser, R., and Novick, P. (1995). The role of Myo2, a yeast class V myosin, in vesicular transport. J. Cell Biol. 128, 1055-1068.

Guertin, D. A., Trautmann, S., and McCollum, D. (2002). Cytokinesis in eukaryotes. Microbiol. Mol. Biol. Rev. 66, 155-178.

Johnston, G. C., Prendergast, J. A., and Singer, R. A. (1991). The Saccharomyces cerevisiae $\mathrm{MYO} 2$ gene encodes an essential myosin for vectorial transport of vesicles. J. Cell Biol. 113, 539-551.

Kiger, A. A., Baum, B., Jones, S., Jones, M. R., Coulson, A., Echeverri, C., and Perrimon, N. (2003). A functional genomic analysis of cell morphology using RNA interference. J. Biol. 2, 27.

Kitayama, C., Sugimoto, A., and Yamamoto, M. (1997). Type II myosin heavy chain encoded by the myo2 gene composes the contractile ring during cytokinesis in Schizosaccharomyces pombe. J. Cell Biol. 137, 1309-1319.

Laevsky, G., and Knecht, D. A. (2003). Cross-linking of actin filaments by myosin-II is a major contributor to cortical integrity and cell motility in restrictive environments. J. Cell Sci. 116, 3761-3770.

Le Goff, X., Motegi, F., Salimova, E., Mabuchi, I., and Simanis, V. (2000). The S. pombe rlc1 gene encodes a putative myosin regulatory light chain that binds the type II myosins myo3p and myo2p. J. Cell Sci. 113, 4157-4163.

Lillie, S. H., and Brown, S. S. (1994). Immunofluorescence localization of the unconventional myosin, Myo2p, and the putative kinesin-related protein, Smylp, to the same regions of polarized growth in Saccharomyces cerevisiae. J. Cell Biol. 125, 825-842.

Lillie, S. H., and Brown, S. S. (1998). Smy1p, a kinesin-related protein that does not require microtubules. J. Cell Biol. 140, 873-883.

Lippincott, J., and Li, R. (1998). Sequential assembly of myosin II, an IQGAPlike protein, and filamentous actin to a ring structure involved in budding yeast cytokinesis. J. Cell Biol. 140, 355-366.
Liu, X., Osherov, N., Yamashita, R., Brzeska, H., Korn, E. D., and May, G. S (2001). Myosin I mutants with only $1 \%$ of wild-type actin-activated MgATPase activity retain essential in vivo function(s). Proc. Natl. Acad. Sci. USA 98, 9122-9127.

Lord, M., and Pollard, T. D. (2004). UCS protein Rng3p activates actin filament gliding by fission yeast myosin-II. J. Cell Biol. 167, 315-325.

Luo, J., Vallen, E. A., Dravis, C., Tcheperegine, S. E., Drees, B., and Bi, E. (2004). Identification and functional analysis of the essential and regulatory light chains of the only type II myosin Myolp in Saccharomyces cerevisiae. J. Cell Biol. 165, 843-855.

Lupas, A., Van Dyke, M., and Stock, J. (1991). Predicting coiled coils from protein sequences. Science 252, 1162-1164.

Mabuchi, I., and Okuno, M. (1977). The effect of myosin antibody on the division of starfish blastomeres. J. Cell Biol. 74, 251-263.

May, K. M., Watts, F. Z., Jones, N., and Hyams, J. S. (1997). Type II myosin involved in cytokinesis in the fission yeast, Schizosaccharomyces pombe. Cell Motil. Cytoskeleton 38, 385-396.

McCollum, D., Balasubramanian, M. K., Pelcher, L. E., Hemmingsen, S. M., and Gould, K. L. (1995). Schizosaccharomyces pombe cdc4+ gene encodes a novel EF-hand protein essential for cytokinesis. J. Cell Biol. 130, 651-660.

Moreno, S., Klar, A., and Nurse, P. (1991). Molecular genetic analysis of fission yeast Schizosaccharomyces pombe. Methods Enzymol. 194, 795-823.

Motegi, F., Mishra, M., Balasubramanian, M. K., and Mabuchi, I. (2004). Myosin-II reorganization during mitosis is controlled temporally by its dephosphorylation and spatially by Mid1 in fission yeast. J. Cell Biol. 165, 685-695.

Motegi, F., Nakano, K., Kitayama, C., Yamamoto, M., and Mabuchi, I. (1997). Identification of Myo3, a second type-II myosin heavy chain in the fission yeast Schizosaccharomyces pombe. FEBS Lett. 420, 161-166.

Motegi, F., Nakano, K., and Mabuchi, I. (2000). Molecular mechanism of myosin-II assembly at the division site in Schizosaccharomyces pombe. J. Cell Sci. 113, 1813-1825

Mulvihill, D. P., Barretto, C., and Hyams, J. S. (2001). Localization of fission yeast type II myosin, $\mathrm{Myo}$, to the cytokinetic actin ring is regulated by phosphorylation of a C-terminal coiled-coil domain and requires a functional septation initiation network. Mol. Biol. Cell 12, 4044-4053.

Mulvihill, D. P., and Hyams, J. S. (2003). Role of the two type II myosins, Myo2 and Myp2, in cytokinetic actomyosin ring formation and function in fission yeast. Cell Motil. Cytoskeleton 54, 208-216.

Naqvi, N. I., Eng, K., Gould, K. L., and Balasubramanian, M. K. (1999). Evidence for F-actin-dependent and -independent mechanisms involved in assembly and stability of the medial actomyosin ring in fission yeast. EMBO J. $18,854-862$.

Naqvi, N. I., Wong, K. C., Tang, X., and Balasubramanian, M. K. (2000). Type II myosin regulatory light chain relieves auto-inhibition of myosin-heavychain function. Nat. Cell Biol. 2, 855-858.

Pasternak, C., Spudich, J. A., and Elson, E. L. (1989). Capping of surface receptors and concomitant cortical tension are generated by conventional myosin. Nature 341, 549-551.

Rogers, S. L., Wiedemann, U., Stuurman, N., and Vale, R. D. (2003). Molecular requirements for actin-based lamella formation in Drosophila S2 cells. J. Cell Biol. 162, 1079-1088.

Rose, M. D., Winston, F., and Heiter, P. (1990). Methods in Yeast Genetics, Cold Spring Harbor, NY: Cold Spring Harbor Laboratory Press.

Schmidt, M., Bowers, B., Varma, A., Roh, D. H., and Cabib, E. (2002). In budding yeast, contraction of the actomyosin ring and formation of the primary septum at cytokinesis depend on each other. J. Cell Sci. 115, 293-302.

Sellers, J. R., Pato, M. D., and Adelstein, R. S. (1981). Reversible phosphorylation of smooth muscle myosin, heavy meromyosin and platelet myosin. J. Biol. Chem. 256, 13137-13142.

Shu, S., Lee, R. J., LeBlanc-Straceski, J. M., and Uyeda, T. Q. (1999). Role of myosin II tail sequences in its function and localization at the cleavage furrow in Dictyostelium J. Cell Sci. 112, 2195-2201.

Shu, S., Liu, X., and Korn, E. D. (2003). Dictyostelium and Acanthamoeba myosin II assembly domains go to the cleavage furrow of Dictyostelium myosin II-null cells. Proc. Natl. Acad. Sci. USA 100, 6499-6504.

Sikorski, R. S., and Hieter, P. (1989). A system of shuttle vectors and yeast host strains designed for efficient manipulation of DNA in Saccharomyces cerevisiae. Genetics 122, 19-27.

Somma, M. P., Fasulo, B., Cenci, G., Cundari, E., and Gatti, M. (2002). Molecular dissection of cytokinesis by RNA interference in Drosophila cultured cells. Mol. Biol. Cell 13, 2448-2460. 
Toi, H., Fujimura-Kamada, K., Irie, K., Takai, Y., Todo, S., and Tanaka, K. (2003). She4p/Dim1p interacts with the motor domain of unconventional myosins in the budding yeast, Saccharomyces cerevisiae Mol. Biol. Cell 14, 2237-2249.

Tolliday, N., Pitcher, M., and Li, R. (2003). Direct evidence for a critical role of myosin II in budding yeast cytokinesis and the evolvability of new cytokinetic mechanisms in the absence of myosin II. Mol. Biol. Cell 14, 798-809.

VerPlank, L., and Li, R. (2005). Cell cycle-regulated trafficking of Chs2 controls actomyosin ring stability during cytokinesis. Mol. Biol. Cell 16, 2529-2543.

Wagner, W., Bielli, P., Wacha, S., and Ragnini-Wilson, A. (2002). Mlc1p promotes septum closure during cytokinesis via the IQ motifs of the vesicle motor Myo2p. EMBO J. 21, 6397-6408.

Watts, F. Z., Shiels, G., and Orr, E. (1987). The yeast MYO1 gene encoding a myosin-like protein required for cell division. EMBO J. 6, 3499-3505.

Wesche, S., Arnold, M., and Jansen, R. P. (2003). The UCS domain protein She $4 p$ binds to myosin motor domains and is essential for class I and class V myosin function. Curr. Biol. 13, 715-724.
Wong, K. C., Naqvi, N. I., Iino, Y., Yamamoto, M., and Balasubramanian, M. K. (2000). Fission yeast Rng3p: an UCS-domain protein that mediates myosin II assembly during cytokinesis. J. Cell Sci. 113, 2421-2432.

Wu, J. Q., Kuhn, J. R., Kovar, D. R., and Pollard, T. D. (2003). Spatial and temporal pathway for assembly and constriction of the contractile ring in fission yeast cytokinesis. Dev. Cell 5, 723-734.

Xu, X. S., Lee, E., Chen, T., Kuczmarski, E., Chisholm, R. L., and Knecht, D. A. (2001). During multicellular migration, myosin II serves a structural role independent of its motor function. Dev. Biol. 232, 255-264.

Yumura, S., and Uyeda, T. Q. (1997). Transport of myosin II to the equatorial region without its own motor activity in mitotic Dictyostelium cells. Mol. Biol. Cell 8, 2089-2099.

Zang, J. H., and Spudich, J. A. (1998). Myosin II localization during cytokinesis occurs by a mechanism that does not require its motor domain. Proc. Natl. Acad. Sci. USA 95, 13652-13657. 